\title{
ON QUANTIZABLE ODD LIE BIALGEBRAS
}

\author{
ANTON KHOROSHKIN, SERGEI MERKULOV, AND THOMAS WILLWACHER
}

\begin{abstract}
Motivated by the obstruction to the deformation quantization of Poisson structures in infinite dimensions we introduce the notion of a quantizable odd Lie bialgebra. The main result of the paper is a construction of the highly non-trivial minimal resolution of the properad governing such Lie bialgebras, and its link with the theory of so called quantizable Poisson structures.
\end{abstract}

\section{INTRODUCTION}

1.1. Even and odd Lie bialgebras. A Lie $(c, d)$-bialgebra is a graded vector space $V$ which carries both a degree $c$ Lie algebra structure

$$
\stackrel{1}{1}_{2}^{1} \simeq[,]: V \otimes V \rightarrow V[c]
$$

and a degree $d$ Lie coalgebra structure,

$$
{ }_{1}^{1} \Upsilon^{2} \simeq \Delta: V \rightarrow V \otimes V[d]
$$

satisfying the following compatibility condition:

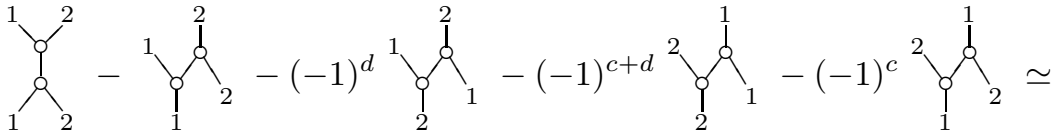

$$
\begin{aligned}
& \simeq \Delta\left(\left[x_{1}, x_{2}\right]\right)-\left[\Delta\left(x_{1}\right), x_{2} \otimes 1+1 \otimes x_{2}\right] \pm\left[x_{1} \otimes 1+1 \otimes x_{1}, \Delta\left(x_{2}\right)\right]=0 .
\end{aligned}
$$

If the $\mathbb{Z}_{2}$-parities of both structures are the same, i.e. if $c+d \in 2 \mathbb{Z}$, the Lie bialgebra is called even, if the $\mathbb{Z}_{2}$-parities are opposite, $c+d \in 2 \mathbb{Z}+1$, it is called odd.

In the even case the most interesting for applications Lie bialgebras have $c=d=0$. Such Lie bialgebras were introduced by Drinfeld in [D] in the context of the theory of Yang-Baxter equations, and they have since found numerous applications, most prominently in the theory of Hopf algebra deformations of universal enveloping algebras (see the book [ES] and references cited there). If the composition of the cobracket and bracket of a Lie bialgebra is zero, that is

$$
\stackrel{b}{<}>=0
$$

then the Lie bialgebra is called involutive. This additional constraint is satisfied in many interesting examples studied in homological algebra, string topology, symplectic field theory, Lagrangian Floer theory of higher genus, and the theory of cohomology groups $H\left(\mathcal{M}_{g, n}\right)$ of moduli spaces of algebraic curves with labelings of punctures skewsymmetrized [D, ES, C, CS, CFL, Sc, CMW, MW1].

In the odd case the most interesting for applications Lie bialgebras have $c=1, d=0$. They have been introduced in M1 and have seen applications in Poisson geometry, deformation quantization of Poisson structures [M2] and in the theory of cohomology groups $H\left(\mathcal{M}_{g, n}\right)$ of moduli spaces of algebraic curves with labelings of punctures symmetrized [MW1.

The homotopy and deformation theories of even/odd Lie bialgebras and also of involutive Lie bialgebras have been studied in CMW, MW2. A key tool in those studies is a minimal resolution of the properad governing the algebraic structure under consideration.

The minimal resolutions of properads $\mathcal{L} i e b$ and $\mathcal{L} i e b_{\text {odd }}$ governing even and, respectively, odd Lie bialgebras were constructed in [Ko, MaVo, V] and, respectively, in [M1, M2]. Constructing a minimal resolution $\mathcal{H}$ olieb $^{\diamond}$ 
of the properad $\mathcal{L} i e b^{\diamond}$ governing involutive Lie bialgebras turned out to be a more difficult problem, and that goal was achieved only very recently in CMW.

1.2. Quantizable odd Lie bialgebras. For odd Lie bialgebras the involutivity condition (1) is trivial, i.e. it is satisfied automatically for any odd Lie bialgebra $V$. There is, however, a higher genus analogue of that condition,

$$
\sum_{0}^{1}=0 \text {, }
$$

which is highly non-trivial, and which can be considered as an odd analogue of (1). We prefer, however, to call odd Lie bialgebras satisfying the extra constraint (2) quantizable ones rather than involutive. The reason for this terminology is explained in $\S 2$ : this graph controls, on the one hand, the obstruction to the universal quantization of Poisson structures in infinite dimensions [M1, M2, and, on the other hand, it controls the obstruction to the existence of geometrically meaningful Frobenius structure on Chains $(\mathbb{R})[\mathrm{JF}$.

Our main result is an explicit construction in $\S 3$ of a (highly non-obvious) minimal resolution $\mathcal{H}_{\text {olieb }}^{\diamond}$ odd of the properad $\mathcal{L} i e b_{\text {odd }}^{\diamond}$ governing quantizable Lie bialgebras. One of the key tricks in [CMW] used to solve an analogous problem for the properad $\mathcal{L} i e b^{\diamond}$ of even involutive Lie bialgebras reduced the "hard" problem of computing the cohomology of some dg properad to the "easy" computation of the minimal resolutions of a family of some auxiliary quadratic algebras. Remarkable enough, this approach works for the constraint (2) as well, but it leads instead to a certain family of cubic homogeneous algebras which are studied in the Appendix.

Another important technical ingredient in our construction of $\mathcal{H}$ olieb ${ }_{\text {odd }}^{\diamond}$ comes from the paper [MW2, in which the cohomologies of the deformation complexes of the properads $\mathcal{L} i e b, \mathcal{L}_{i e b_{\text {odd }}}$ and $\mathcal{L} i e b^{\diamond}$ have been computed, and it was proven in particular that the properad $\mathcal{L} i e b_{\text {odd }}$ admits precisely one non-trivial deformation; in fact it is that unique non-trivial deformation which leads us to the dg properad $\mathcal{H}$ olieb $b_{\text {odd }}^{\diamond}$. We explain this link in $\S 3$.

There are plenty of examples of $\mathcal{H}$ olie $b_{\text {odd }}^{\diamond}$ algebra structures associated with ordinary (or formal power series) Poisson structures $\pi \in \mathcal{T}_{\text {poly }}\left(\mathbb{R}^{d}\right)$ on $\mathbb{R}^{d}, d \geq 2$, which vanish at $0 \in \mathbb{R}^{d}$; for a generic Poisson structure $\pi$ on $\mathbb{R}^{d}$ the associated $\mathcal{H}$ olieb ${ }_{\text {odd }}^{\diamond}$ algebra structure $\pi^{\diamond}$,

$$
\pi^{\diamond}: \mathcal{H} \text { olieb } b_{\text {odd }}^{\diamond} \longrightarrow \mathcal{E} n d_{\mathbb{R}^{d}}
$$

in $\mathbb{R}^{d}$ is highly non-trivial and can be given explicitly only by transcendental formulae (i.e. the ones involving converging integrals over suitable configuration spaces [MW3]). Such a $\mathcal{H}$ olieb $b_{\text {odd }}^{\diamond}$ algebra structure $\pi^{\diamond}$ in $\mathbb{R}^{d}$ can also be interpreted as a formal power series bivector field $\pi^{\diamond} \in \mathcal{T}_{\text {poly }}\left(\mathbb{R}^{d}\right)[[\hbar]]$,

$$
\pi^{\diamond}=\pi_{0}+\hbar \pi_{1}+\hbar^{3} \pi_{2}+\ldots,
$$

satisfying a certain formal power series equation,

$$
\frac{1}{2}\left[\pi^{\diamond}, \pi^{\diamond}\right]_{2}+\frac{\hbar}{4 !}\left[\pi^{\diamond}, \pi^{\diamond}, \pi^{\diamond}, \pi^{\diamond}\right]_{4}+\ldots=0,
$$

Here the collection of operators,

$$
\left\{[, \ldots,]_{2 n}: \mathcal{T}_{\text {poly }}\left(\mathbb{R}^{d}\right)^{\otimes 2 n} \rightarrow \mathcal{T}_{\text {poly }}\left(\mathbb{R}^{d}\right)[3-4 n]\right\}_{n \geq 1}
$$

defines a so called Kontsevich-Shoikhet $\mathcal{L} i e_{\infty}$ structure $[$ Sh $]$ in $\mathcal{T}_{\text {poly }}\left(\mathbb{R}^{d}\right)$ with $[,]_{2}$ being the standard Schouten bracket. This huge class $\left\{\pi^{\diamond}\right\}$ of highly-non-trivial examples of $\mathcal{H}$ olieb $b_{\text {odd }}^{\diamond}$ algebra structures in $\mathbb{R}^{d}$ depends in general on the choice of Drinfeld associator [MW3]; it motivated much our current study of the homotopy theory of odd Lie bialgebras.

1.3. Some notation. The set $\{1,2, \ldots, n\}$ is abbreviated to $[n]$; its group of automorphisms is denoted by $\mathbb{S}_{n}$; the trivial one-dimensional representation of $\mathbb{S}_{n}$ is denoted by $\mathbf{1}_{n}$, while its one dimensional sign representation is denoted by $s g n_{n}$. The cardinality of a finite set $A$ is denoted by $\# A$.

We work throughout in the category of $\mathbb{Z}$-graded vector spaces over a field $\mathbb{K}$ of characteristic zero. If $V=\oplus_{i \in \mathbb{Z}} V^{i}$ is a graded vector space, then $V[k]$ stands for the graded vector space with $V[k]^{i}:=V^{i+k}$. For 
a prop(erad) $\mathcal{P}$ we denote by $\mathcal{P}\{k\}$ a prop(erad) which is uniquely defined by the following property: for any graded vector space $V$ a representation of $\mathcal{P}\{k\}$ in $V$ is identical to a representation of $\mathcal{P}$ in $V[k]$.

Acknowledgements. We are very grateful to Jan-Erik Roos for valuable correspondences concerning Koszulness theory of homogeneous algebras and to Dmitri Piontkovski for inspiring discussions on algebras of homological dimension 2 and strongly free Gröbner bases.

A.Kh. acknowledges partial support by RFBR grant 15-01-09242, by Dynasty foundation and Simons-IUM fellowship, partially prepared within the framework of a subsidy granted to the National Research University Higher School of Economics by the Government of the Russian Federation for the implementation of the Global Competitiveness Program. S.M. acknowledges support by the Swedish Vetenskaprådet, grant 20125478. T.W. acknowledges partial support by the Swiss National Science Foundation, grant 200021_150012, and by the NCCR SwissMAP of the Swiss National Science Foundation.

\section{Quantizable odd Lie bialgebras}

2.1. Odd lie bialgebras. By definition [M1, the properad, Lieb $\diamond_{\text {odd }}$, of odd Lie bialgebras is a quadratic properad given as the quotient,

$$
\mathcal{L} i e b_{\text {odd }}^{\diamond}:=\mathcal{F} \text { ree }\langle E\rangle /\langle\mathcal{R}\rangle,
$$

of the free properad generated by an $\mathbb{S}$-bimodule $E=\{E(m, n)\}_{m, n \geq 1}$ with all $E(m, n)=0$ except

$$
\begin{aligned}
& E(2,1):=\operatorname{sgn}_{2} \otimes \mathbf{1}_{1}=\operatorname{span}\left\langle{ }^{1} Y_{1}^{2}=-\Upsilon_{1}^{2}{ }^{1}\right\rangle
\end{aligned}
$$

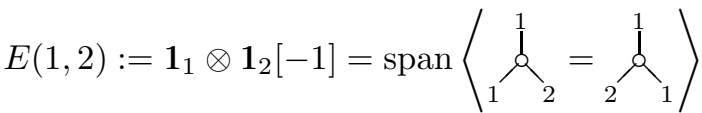

modulo the ideal generated by the following relations

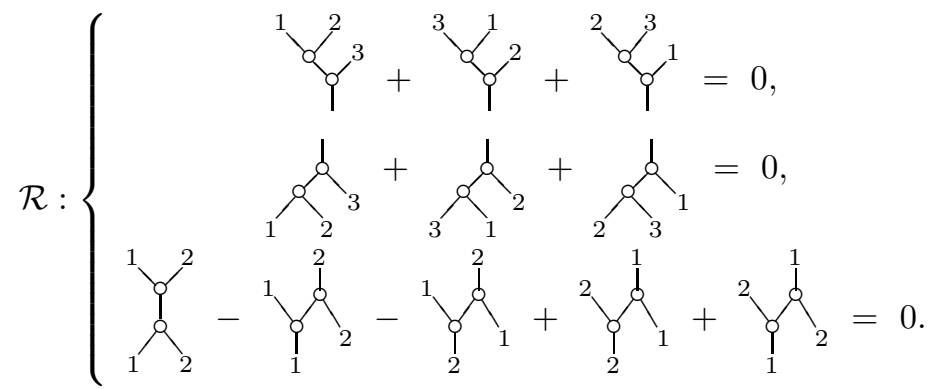

A minimal resolution $\mathcal{H}_{\text {olieb }}$ odd of $\mathcal{L}_{2} e b_{\text {odd }}$ was constructed in [M1, M2]. It is a free properad,

$$
\mathcal{H}_{\text {olieb }} \text { odd }=\mathcal{F} \text { ree }\langle\hat{E}\rangle
$$

generated by an $\mathbb{S}$-bimodule $\hat{E}=\{\hat{E}(m, n)\}_{m, n \geq 1, m+n \geq 3}$,

$$
\hat{E}(m, n):=\operatorname{sgn}_{m} \otimes \mathbf{1}_{n}[m-2]=\operatorname{span}\left\langle\sum_{2}^{1}\right.
$$

and comes equipped with the differential



It was shown in [M1, M2] that representations $\mathcal{H}$ olieb $_{\text {odd }} \rightarrow \mathcal{E} n d_{V}$ of the minimal resolution of $\mathcal{L}_{\text {ieb }}$ odd in a graded vector space $V$ are in 1-1 correspondence with formal graded Poisson structures $\pi \in \mathcal{T}_{\text {poly }}^{\geq 1}\left(V^{*}\right)$ on the dual vector space $V^{*}$ (viewed as a linear manifold) which vanish at the zero point in $V,\left.\pi\right|_{0}=0$. 
2.2. Quantizable odd Lie bialgebras. We define the properad $\mathcal{L} i e b_{\text {odd }}^{\diamond}$ of quantizable odd Lie bialgebras as the quotient of the properad $\mathcal{L}_{i e b_{\text {odd }}}$ by the ideal generated by the following element



The associated relation on Lie and coLie brackets looks like a higher genus odd analogue of the involutivity condition (1) in the case of even Lie bialgebras. However, we prefer to use the adjective quantizable rather than involutive for odd Lie bialgebras satisfying (2) because that condition has a clear interpretation within the framework of the theory of deformation quantization, and its quantizability property becomes even more clear when one raises it to the level of representations of its minimal resolution $\mathcal{H}$ olieb odd.

An odd Lie bialgebra structure in a vector space $V$ can be understood as a pair

$$
\left(\xi \in \mathcal{T}_{V^{*}}, \Phi \in \wedge^{2} \mathcal{T}_{V^{*}}\right)
$$

consisting of a degree 1 quadratic vector field $\xi$ (corresponding to the Lie cobracket $\Delta$ in $V$ ) and a linear Poisson structure $\Phi$ in $V^{*}$ (corresponding to the Lie bracket [, ] in $V$ ). All the (compatibility) equations for the algebraic operations [, ] and $\Delta$ get encoded into a single equation,

$$
\{\xi+\Phi, \xi+\Phi\}=0
$$

where $\{$,$\} stand for the standard Schouten bracket in the algebra \mathcal{T}_{\text {poly }}\left(V^{*}\right)$ of polyvector fields on $V^{*}$ (viewed as an affine manifold). Therefore, the sum $\xi+\Phi$ gives us a graded Poisson structure on $V^{*}$ and one can talk about its deformation quantization, that is, about an associated Maurer-Cartan element $\Gamma$ (deforming $\xi+\Phi)$ in the Hochschild dg Lie algebra,

$$
C^{\bullet}\left(\mathcal{O}_{V}, \mathcal{O}_{V}\right):=\bigoplus_{n \geq 0} \operatorname{Hom}\left(\mathcal{O}_{V}^{\otimes n}, \mathcal{O}_{V}\right)
$$

where $\operatorname{Hom}\left(\mathcal{O}_{V}^{\otimes n}, \mathcal{O}_{V}\right)$ stands for the vector space of polydifferential operators on the graded commutative algebra $\mathcal{O}_{V}:=\odot V$ of polynomial functions on $V^{*}$. As the graded Poisson structure $\xi+\Phi$ is non-negatively graded, its deformation quantization must satisfy the condition

$$
\Gamma \in \operatorname{Hom}\left(\mathbb{K}, \mathcal{O}_{V}\right) \oplus \operatorname{Hom}\left(\mathcal{O}_{V}, \mathcal{O}_{V}\right) \oplus \operatorname{Hom}\left(\mathcal{O}_{V}^{\otimes 2}, \mathcal{O}_{V}\right)
$$

with the corresponding splitting of $\Gamma$ into a sum of three terms,

$$
\Gamma=\Gamma_{0}+\Gamma_{1}+\Gamma_{2}
$$

of degrees 2, 1 , and 0 respectively. The term $\Gamma_{2}=\Gamma_{2}(\Phi)$ has degree zero and hence can depend (universally) only on the Lie bracket $\Phi$. It makes $\left(\mathcal{O}_{V}, \star:=\Gamma_{2}\right)$ into an associative non-commutative algebra, and up to gauge equivalence, the algebra $\left(\mathcal{O}_{V}, \star\right)$ can always be identified with the universal enveloping algebra of the Lie algebra $(V,[]$,$) . The operation \Gamma_{1}$ is a deformation of the differential on $\mathcal{O}_{V}$ induced by $\xi$. (Note that this latter undeformed differential squares to zero by the second Jacobi identity in the list (3).) The Maurer-Cartan equation for $\Gamma$ states that $\Gamma_{1}$ is a derivation with respect to the star product $\Gamma_{2}$, and squares to zero modulo the star product commutator with the (potential) obstruction $\Gamma_{0}$. Now one can ask whether it is possible to find $\Gamma$ as above such that the obstruction (or sometimes called "curvature") term $\Gamma_{0}$ vanishes, and hence $\Gamma_{1}^{2}=0$, so that $\Gamma_{1}$ is an honest differential. Since the algebra $\left(\mathcal{O}_{V}, \star\right)$ is generated by $V$, any derivation with respect to the product $\star$ is uniquely determined by its values on $V$. Let $\xi_{\star}$ be the unique derivation of the associative algebra $\left(\mathcal{O}_{V}, \star\right)$ that agrees with $\xi$ on $V$. This derivation is well defined since it annihilates the defining relations of the universal enveloping algebra by the third relation in (3). Then the derivation $\xi_{\star}^{2}=\frac{1}{2}\left[\xi_{\star}, \xi_{\star}\right]=0$ if and only if $\xi \simeq \mathcal{l}$ and $\Phi \simeq \zeta$ satisfy the extra compatibility condition (2) (see [M3]). Therefore, if $\xi$ and $\Phi$ come from a representation of $\mathcal{L} i e b_{\text {odd }}^{\diamond}$ in $V$, then they admit a very simple deformation quantization in the form

$$
\Gamma=\xi_{\star}+\Gamma_{2}(\Phi),
$$

and this quantization makes sense even in the case when $V$ is infinite-dimensional.

If $\xi$ and $\Phi$ do not satisfy the extra compatibility condition (2), then their deformation quantization is possible only in finite dimensions, and involve a non-zero "curvature" term $\Gamma_{0}$ which in turn involves graphs with 
closed paths of directed edges and is given explicitly in [M3 (in fact this argument proves non-existence of Kontsevich formality maps for infinite dimensional manifolds).

These considerations shall motivate our notation "quantizable Lie bialgebras" for odd Lie bialgebras satisfying the condition (2).

We shall construct below a minimal resolution $\mathcal{H}$ olieb $b_{\text {odd }}^{\diamond}$ of the properad $\mathcal{L}$ ieb $b_{\text {odd }}^{\diamond}$; its representations in a graded vector space $V$ give us so called quantizable Poisson structures on $V$ which can be deformation quantized via a trivial (i.e. without using Drinfeld associators) perturbation even if $\operatorname{dim} V=\infty$ (see W2, B]); in finite dimensions there is a 1-1 correspondence between ordinary Poisson structure on $V$ and quantizable ones, but this correspondence is highly non-trivial - it depends on the choice of a Drinfeld associator [MW3]. The properad Koszul dual to the properad $\mathcal{L}_{i e b_{o d d}}$ is the properad of odd Frobenius algebras (cf. [V]). A remarkable "Koszul dual" meaning of the graph (2) was found by Theo Johnson-Freyd in [JF — it controls the obstruction to the existence of a geometrically meaningful homotopy odd Frobenius structure on the complex Chains. $(\mathbb{R})$.

\section{A minimal resolution of $\mathcal{L} i e b_{\text {odd }}^{\diamond}$}

3.1. Oriented graph complexes and a Kontsevich-Shoikhet MC element. Let $G_{n, l}^{o r}$ be a set of connected graphs $\Gamma$ with $n$ vertices and $l$ directed edges such that (i) $\Gamma$ has no closed directed paths of edges, and (ii) some bijection from the set of edges $E(\Gamma)$ to the set $[l]$ is fixed. There is a natural right action of the group $\mathbb{S}_{l}$ on the set $G_{n, l}^{o r}$ by relabeling the edges.

Consider a graded vector space

$$
\mathrm{fGC}_{2}^{o r}:=\prod_{n \geq 1, l \geq 0} \mathbb{K}\left\langle G_{n, l}^{o r}\right\rangle \otimes_{\mathbb{S}_{l}} \operatorname{sgn}_{l}[l+2(1-n)]
$$

It was shown in W2 that this vector space comes equipped with a Lie bracket [, ] (given, as often in the theory of graph complexes, by substituting graphs into vertices of another graphs), and that the degree +1 graph

$$
\bullet \rightarrow \bullet \in \mathrm{fGC}_{2}^{\text {or }}
$$

is a Maurer-Cartan element making $\mathrm{fGC}_{2}^{\text {or }}$ into a differential Lie algebra with the differential given by

$$
\delta:=[\bullet \rightarrow \bullet,] .
$$

It was proven in $\mathrm{W} 2$ that the cohomology group $H^{1}\left(\mathrm{fGC}_{2}^{\text {or }}\right)$ is one-dimensional and is spanned by the following graph

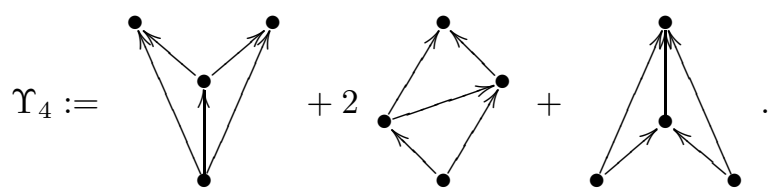

while the cohomology group $H^{2}\left(\mathrm{fGC}_{2}^{\text {or }}, \delta_{0}\right)$ is also one-dimensional and is generated by a linear combination of graphs with four vertices (whose explicit form plays no role in this paper). This means that one can construct by induction a new Maurer-Cartan element (the integer subscript stand for the number of vertices)

$$
\Upsilon_{K S}=\bullet \rightarrow \bullet+\Upsilon_{4}+\Upsilon_{6}+\Upsilon_{8}+\ldots
$$

in the Lie algebra $\mathrm{fGC}_{2}^{o r}$. Indeed, the Lie brackets in $\mathrm{fGC}_{2}^{o r}$ has the property that a commutator $[A, B]$ of a graph $A$ with $p$ vertices and a graph $B$ with $q$ vertices has $p+q-1$ vertices. Therefore, all the obstructions to extending the sum $\bullet \longrightarrow \bullet+\Upsilon_{4}$ to a Maurer-Cartan element have 7 or more vertices and hence do not hit the unique cohomology class in $H^{2}\left(\mathrm{fGC}_{2}^{o r}, \delta\right)$. Up to gauge equivalence, this new MC element $\Upsilon_{K S}$ is the only non-trivial deformation of the standard MC element $\bullet \longrightarrow \bullet$. We call it the Kontsevich-Shoikhet element as it was introduced (via a different line of thought) by Boris Shoikhet in Sh with a reference to an important contribution by Maxim Kontsevich via an informal communication. 
3.1.1. A formal power series extension of $\mathrm{fGC}_{2}^{\text {or }}$. Let $\hbar$ be a formal parameter of degree 0 and let $\mathrm{fGC}_{2}^{o r}[[\hbar]]$ be a topological vector space of formal power series in $\hbar$ with coefficients in $\mathrm{fGC}_{2}^{\text {or }}$. This is naturally a topological Lie algebra in which the formal power series

$$
\Upsilon_{K S}^{\hbar}=\bullet \rightarrow \bullet+\hbar \Upsilon_{4}++\hbar^{2} \Upsilon_{6}+\hbar^{3} \Upsilon_{8}+\ldots
$$

is a Maurer-Cartan element.

3.2. From the Kontsevich-Shoikhet element to a minimal resolution of $\mathcal{L} i e b_{\text {odd }}^{\diamond}$. Consider a (nondifferential) free properad $\mathcal{H}$ olieb $b_{\text {odd }}^{\diamond}$ generated by the following (skewsymmetric in outputs and symmetric in inputs) corollas of degree $2-m$,

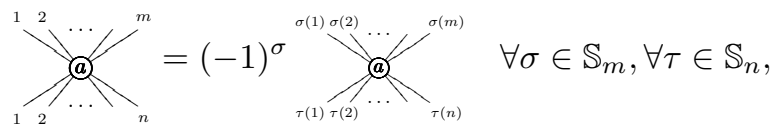

where $m+n+a \geq 3, m \geq 1, n \geq 1, a \geq 0$. Let $\widehat{\mathcal{H} \text { olieb }} b_{\text {odd }}^{\diamond}$ be the genus completion of $\mathcal{H}_{\text {olie }} b_{\text {odd }}$.

3.2.1. Lemma. The Lie algebra $\mathrm{fGC}_{2}^{\text {or }}[[\hbar]]$ acts (from the right) on the properad $\widehat{\mathcal{H}}{ }^{\circ}{ }^{\diamond} b_{\text {odd }}$ by continuous derivations, that is, there is a morphism of Lie algebras

$$
\begin{array}{ccc}
F: \mathrm{fGC}_{2}^{\text {or }}[[\hbar]] & \longrightarrow & \operatorname{Der}\left(\widehat{\mathcal{H} \text { olieb }}_{\text {odd }}^{\diamond}\right) \\
\hbar^{k} \Gamma & \longrightarrow\left(\hbar^{k} \Gamma\right)
\end{array}
$$

where the derivation $F\left(\hbar^{k} \Gamma\right)$ is given on the generators as follows

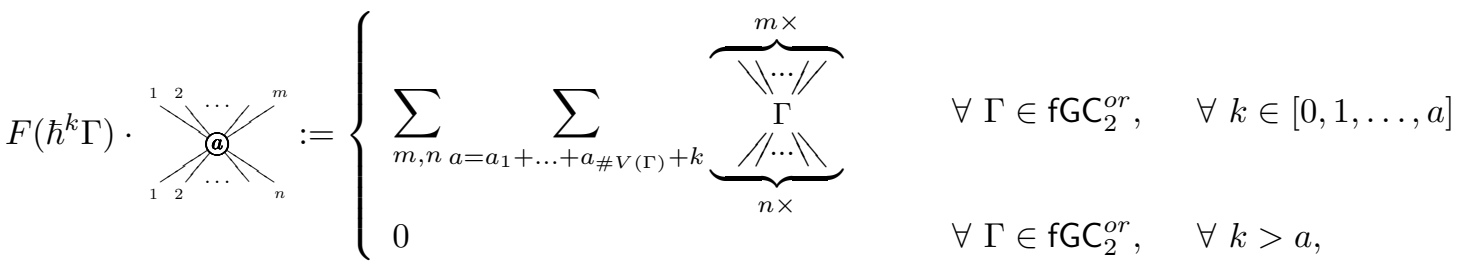

where the first sum is taking over to attach $m$ output legs and $n$ input legs to the vertices of the graph $\Gamma$, and the second sum is taken over all possible ways to decorate the vertices of $\Gamma$ with non-negative integers $a_{1}, \ldots, a_{\# V(\Gamma)}$ such they sum to $a-k$.

Proof is identical to the proofs of similar statements (Theorems 1.2.1 and 1.2.2) in MW2.

3.2.2. Corollary. The completed free properad $\widehat{\mathcal{H o l i e}}_{\text {odd }}^{\diamond}$ comes equipped with a differential $\delta_{\diamond}:=F\left(\Upsilon_{K S}^{\hbar}\right)$. The differential $\delta$ restricts to a differential in the free properad $\mathcal{H}$ olieb ${ }_{\text {odd }}^{\diamond}$.

Proof. When applied to any generator of $\widehat{\mathcal{H o l i e}}_{\text {odd }}^{\diamond}$ the differential $\delta$ gives always a finite sum of graphs. It follows that it is well defined in $\mathcal{H}$ olieb $b_{\text {odd }}^{\diamond}$ as well.

There is an injection of $\mathrm{dg}$ free properads

$$
\left(\mathcal{H}_{\text {olieb }} \text { odd }, \delta\right) \longrightarrow\left(\mathcal{H} \text { olieb }{ }_{\text {odd }}^{\diamond}, \delta_{\diamond}\right)
$$

given on generators by

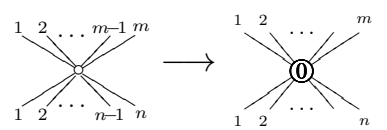

Identifying from now on weight zero generators of $\mathcal{H}_{\text {olieb }}$ odd with generators of $\mathcal{H}_{\text {olieb }}$ odd , we may write

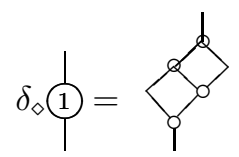


and hence conclude that there is a natural morphism of dg properads

$$
\pi:\left(\mathcal{H} \text { olieb }{ }_{\text {odd }}^{\diamond}, \delta_{\diamond}\right) \longrightarrow\left(\mathcal{L}_{i e} b_{\text {odd }}^{\diamond}, 0\right)
$$

Our main result in this paper is the following theorem.

3.3. Main Theorem. The map $\pi$ is a quasi-isomorphism, i.e. $\mathcal{H}$ olieb ${ }_{\text {odd }}^{\diamond}$ is a minimal resolution of $\mathcal{L}_{\text {ieb }}^{\diamond}$ odd. Proof. Let $\mathcal{P}$ be a dg properad generated by a degree 1 corollas $\phi_{q}$ and $\ell_{1} \ell_{2}=\ell_{2}$, and a degree zero corolla, ${ }^{1} \gamma^{2}=-{ }^{2} Y^{1}$ and modulo relations

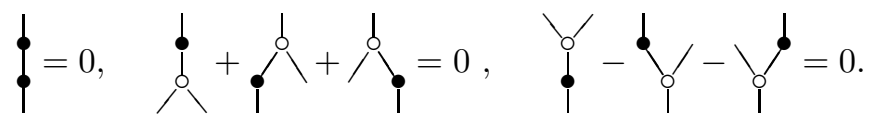

and the three relations in (3). The differential in $\mathcal{P}$ is given on the generators by

$$
d \rho=0, \quad d \%=0, \quad d \phi=\sum_{\varphi}^{\phi}
$$

Claim I: The surjective morphism of $d g$ properads,

$$
\nu: \mathcal{H} \text { olieb } b_{\text {odd }}^{\diamond} \longrightarrow \mathcal{P},
$$

which sends all generators to zero except for the following ones

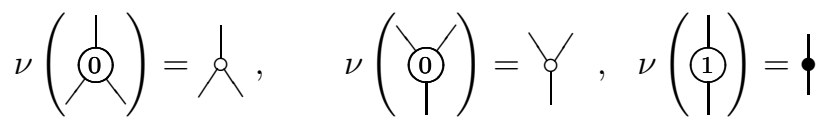

is a quasi-isomorphism.

The proof of this claim is identical to the proof of Theorem 2.7.1 in [CMW] so that we can omit the details.

The proof of the Main Theorem will be completed once we show the following

ClAIM II: The natural map

$$
\mu:(\mathcal{P}, d) \longrightarrow\left(\mathcal{L}_{i e b_{\text {odd }}^{\diamond}}, 0\right)
$$

is a quasi-isomorphism.

Let us define a new homological grading in the properad $\mathcal{P}$ by assigning to the generator $\emptyset$ degree -1 and to the remaining generators the degree zero; to avoid confusion with the original grading let us call this new grading s-grading. Then Claim II is proven once we show that the cohomology $H(\mathcal{P})$ of $\mathcal{P}$ is concentrated in $s$-degree zero.

Consider a path filtration $[\mathrm{Ko}, \mathrm{MaV0}$ of the $\mathrm{dg}$ properad $\mathcal{P}$. The associated graded gr $\mathcal{P}$ can be identified with dg properad generated by the same corollas $\phi, \lambda_{1} \ell_{2}=\ell_{2} \ell_{1}$ and ${ }^{1} \gamma^{2}=-\gamma^{\prime}$ which are subject to the relations (6), the first two relation in (3) and the following one

$$
\bigcap_{2}^{1}=0
$$

The differential in grP is given by the original formula (7). The ClaIm II is proven once it is shown that the cohomology of the properad $\operatorname{gr} \mathcal{P}$ is concentrated in $s$-degree zero, or equivalently, the cohomology of $\mathrm{dg}$ $\operatorname{prop} \mathcal{U}(\operatorname{gr} \mathcal{P})$ generated by this properad is concentrated in $s$-degree zero (as the universal enveloping functor $\mathcal{U}$ from the category of properads to the category of props is exact).

Consider a free prop $\mathcal{F} r e e\langle E\rangle$ generated by an $\mathbb{S}$-bimodule $E=\{E(m, n)\}$ with all $E(m, n)=0$ except the following ones, 


$$
\begin{aligned}
& E(1,1)=\mathbb{K}[-1]=\operatorname{span}\langle\boldsymbol{\phi}\rangle \\
& E(2,1)=\operatorname{sgn}_{2}=\operatorname{span}\left\langle{ }^{1}=-\Upsilon^{\prime}\right\rangle \\
& E(1,2)=\mathbb{K}\left[\mathbb{S}_{2}\right][-1]=\operatorname{span}\left\langle\underset{1}{\ell_{2}} \boldsymbol{\phi}_{1}\right\rangle
\end{aligned}
$$

We assign to the above generators $s$-degrees $-1,0$ and 0 respectively.

Define next a $\operatorname{dg} \operatorname{prop} \mathcal{A}$ as the quotient of the above free prop $\mathcal{F}$ ree $\langle E\rangle$ by the ideal generated by the relations

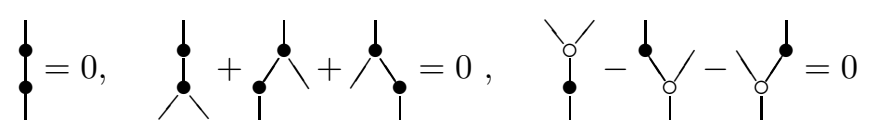

and

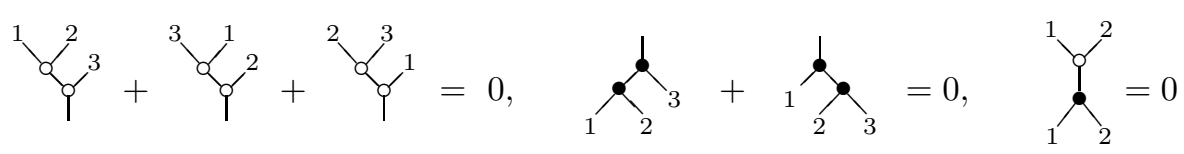

A differential in $\mathcal{A}$ is defined by

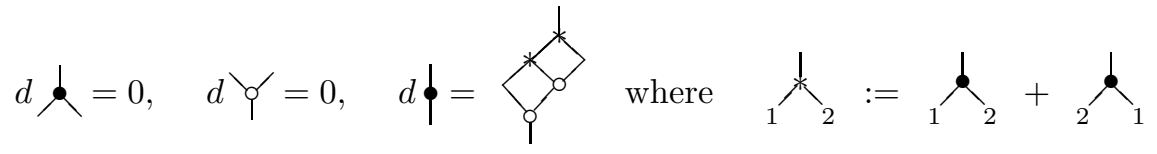

Note that the generator $\downarrow_{1} \star_{2}$ satisfies the second relation in (3) so that we have a canonical injection of dg props

$$
i: \mathcal{U}(\operatorname{gr} \mathcal{P}) \longrightarrow \mathcal{A}
$$

It is easy to see that image of $\mathcal{U}(\operatorname{gr} \mathcal{P})$ under this injection is a direct summand in the complex $(\mathcal{A}, d)$. Hence Claim 2 is proven once we show that the cohomology of the prop $\mathcal{A}$ is concentrated in $s$-degree zero.

Using the associativity relation for the generator $\downarrow$ and the Jacobi relation for the generator $\zeta$ one obtains an equality

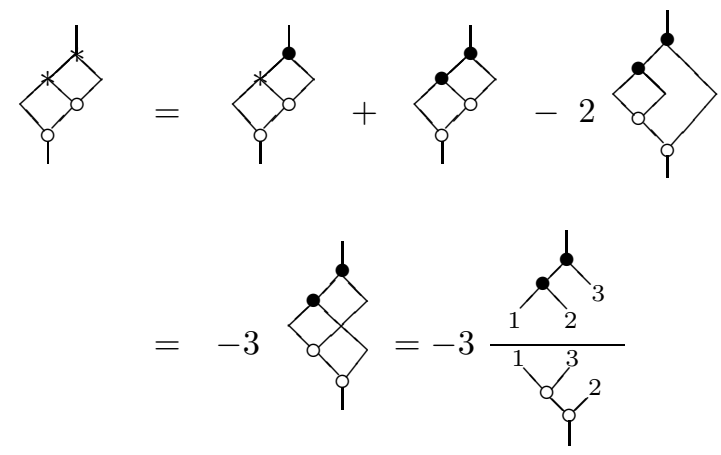

where the horizontal line stands for the properadic composition in accordance with the labels shown.

This result propmts us to consider a dg associative non-commutative algebra $\mathbf{A}_{n}$ generated by degree zero variable $\left\{x_{i}\right\}_{1 \leq i \leq n}$ and degree -1 generators $\left\{u_{i, i+1, u+2}\right\}_{1 \leq i \leq n-2}$ with the differential

$$
d u_{i, i+1, i+2}=-3\left[\left[x_{i}, x_{i+2}\right], x_{i+1}\right]
$$

or, equivalently (after rescaling the generators $u_{\bullet}$ ), with the differential

$$
d u_{i, i+1, i+2}=\underset{8}{\left[\left[x_{i}, x_{i+2}\right], x_{i+1}\right]}
$$


Arguing in exactly the same way as in [CMW] one concludes that the cohomology of the $\operatorname{dg}$ operad operad $\mathcal{A}$ is concentrated in s-degree zero if and only if the collections of algebras $\mathbf{A}_{n}, n \geq 3$, has cohomology concentrated in ordinary degree zero. The latter fact is established in the appendix. The proof is completed.

3.4. Representations of $\mathcal{H}$ lieb $_{\text {odd }}^{\diamond}$ and quantizable Poisson structures. Let $V=\mathbb{R}^{d}$ be a $d$ dimensional vector space, and $\mathcal{O}_{V}=\prod_{n \geq 1} \odot{ }^{n} V^{*}$ the commutative algebra of formal power series functions on $V$, with the obvious complete topology. If $\operatorname{Der}\left(\mathcal{O}_{V}\right)$ stands for the Lie algebra of continuous derivations of $\mathcal{O}_{V}$, then the Lie algebra of formal polyvector fields on $V$ is defined as the Lie algebra of continuous multiderivations,

$$
\mathcal{T}_{\text {poly }}(V):=\widehat{\odot_{\mathcal{O}_{V}}}\left(\operatorname{Der}\left(\mathcal{O}_{V}\right)[1]\right) \cong \prod_{m \geq 0} \wedge^{m} V \otimes \mathcal{O}_{V} \simeq \prod_{m, n \geq 0} \wedge^{m} V \otimes \odot^{n} V^{*}
$$

There is an obvious chain of injections of topological commutative algebras,

$$
\cdots \longrightarrow \mathcal{O}_{\mathbb{R}^{d}} \longrightarrow \mathcal{O}_{\mathbb{R}^{d+1}} \longrightarrow \mathcal{O}_{\mathbb{R}^{d+2}} \longrightarrow \ldots
$$

We denote the associated direct limit by

$$
\mathcal{O}_{\mathbb{R}^{\infty}}:=\lim _{d \rightarrow \infty} \mathcal{O}_{\mathbb{R}^{d}}
$$

For $V=\mathbb{R}^{\infty}$ we define $\mathcal{T}_{\text {poly }}(V)$ as the Lie algebra of continuous multiderivations of $\mathcal{O}_{\mathbb{R}^{\infty}}$, i.e.,

$$
\mathcal{T}_{\text {poly }}(V) \cong \prod_{m \geq 0} \operatorname{Hom}\left(\wedge^{m} \mathbb{R}^{\infty}, \mathcal{O}_{\mathbb{R}}\right)
$$

We can also consider the space $\mathcal{T}_{\text {poly }}(V)[[\hbar]]$ of formal power series in a formal variable $\hbar$ with coefficients in $\mathcal{T}_{\text {poly }}(V)$. These arguments can be easily generalized to a finite/infinite dimensional graded vector space $V$. Consider now a representation of our minimal resolution

$$
\rho: \mathcal{H}_{\text {olieb }} \diamond_{\text {odd }} \longrightarrow \mathcal{E} n d_{V}
$$

in a (possible, infinite-dimensional) dg vector space $V$. It is uniquely determined by the values of $\rho$ on the generators of $\mathcal{H}$ olieb $\diamond_{\text {odd }}^{\diamond}$,

$$
\rho(\left.\overbrace{1}^{1} \underbrace{2}_{n} \ldots\right|^{m}):=\pi_{n}^{m}(a) \in \wedge^{m} V \otimes \odot{ }^{n} V^{*} .
$$

We can assemble these values into a formal power series

$$
\pi^{\diamond}:=\sum_{m, n \geq 0} \sum_{a \geq 0} \hbar^{a} \pi_{n}^{m}(a) \in \mathcal{T}_{\text {poly }}(V)
$$

which gives us a formal polyvector field on $V$. The values $\pi_{n}^{m}(a)$ can not be chosen arbitrarily as the map $\rho$ must respect differentials in $\mathcal{H}_{\text {olieb }}{ }_{\text {odd }}$ and $V$,

$$
\rho \circ \delta_{\diamond}=d \circ \rho .
$$

Untwisting the definition of $\delta_{\diamond}$, we conclude that the above formal power series $\pi^{\diamond}$ (with $\pi_{1}^{1}(0):=d$ ) comes from a representation of $\mathcal{H}$ olieb $_{\text {odd }}^{\diamond}$ if and only if it satisfies the equation

$$
\frac{1}{2}\left[\pi^{\diamond}, \pi^{\diamond}\right]_{2}+\frac{\hbar}{4 !}\left[\pi^{\diamond}, \pi^{\diamond}, \pi^{\diamond}, \pi^{\diamond}\right]_{4}+\ldots=0,
$$

where the collection of operators,

$$
\left\{[, \ldots,]_{2 n}: \mathcal{T}_{\text {poly }}(V)^{\otimes 2 n} \rightarrow \mathcal{T}_{\text {poly }}(V)[3-4 n]\right\}_{n \geq 1}
$$

comes from the values on the graphs $\Upsilon_{2 n}$ from $\S 3.1$ of the standard morphism W1] of dg Lie algebras

$$
\mathrm{fGC}_{2}^{\text {or }} \rightarrow C E^{\bullet}\left(\mathcal{T}_{\text {poly }}(V), \mathcal{T}_{\text {poly }}(V)\right),
$$

$C E^{\bullet}\left(\mathcal{T}_{\text {poly }}(V), \mathcal{T}_{\text {poly }}(V)\right)$ being the Chevalley-Eilenberg deformation complex of the Lie algebra of polyvector fields. Therefore formal quantizable Poisson structures on a graded vector space $V$ (viewed as a formal manifold) come from representations of our properad $\mathcal{H}_{\text {olieb }}^{\diamond}$ odd. There are plenty of examples of such quantizable Poisson structures on finite-dimensional vector spaces, one for each ordinary formal graded 
Poisson structure $\pi$ on $V$ (which is, by definition, an element of $\mathcal{T}_{\text {poly }}(V)$ which satisfies the standard Schouten equation $\left.[\pi, \pi]_{2}=0\right)$. However the association

$$
\pi \longrightarrow \pi^{\diamond}
$$

is highly non-trivial and depends on the choice of a Drinfeld associator [MW3. It is an open problem to find a non-trivial example of a quantizable Poisson structure in infinite dimensions. Perhaps, for any graded vector space $V$ equipped with an odd symplectic form, the associated total space of cyclic words

$$
V:=\Pi_{n \geq 1}\left(\otimes^{n} W\right)_{\mathbb{Z}_{n}}
$$

comes equipped with such a structure given by formulae from Theorem 4.3.3 in [MW1; however it is hard to check this conjecture by a direct computation as it involves infinitely many equations.

\section{Appendix A.}

1.1. Lemma. Let $\left\{c_{\sigma} \mid \sigma \in S_{3}\right\}$ be a collection of 6 numbers such that

for each pair $\left(c_{i j k}, c_{i k j}\right)$ with the same first index $i$ at least one of these elements is different from zero

Then the associative algebra $A:=\mathbb{k}\left\langle x_{1}, \ldots, x_{n} \mid \begin{array}{c}\sum_{\sigma \in S_{3}} c_{\sigma} x_{i+\sigma(1)} x_{i+\sigma(2)} x_{i+\sigma(3)}, \\ i=0, \ldots, n-2\end{array}\right\rangle$ has global dimension 2 .

Proof. Let us consider any linear ordering of the set of generators, such that

$$
\forall k, l, m \quad x_{3 k}>x_{3 l+2}, x_{3 m+1}
$$

We extend this ordering to a degree-lexicographical ordering of the set of monomials in the free associative algebra $\mathbb{k}\left\langle x_{1}, \ldots, x_{n}\right\rangle$. The leading monomials of relation $\sum_{\sigma \in S_{3}} c_{\sigma} x_{i+\sigma(1)} x_{i+\sigma(2)} x_{i+\sigma(3)}$ are different for all $i$ because they contain different letters $\left\{x_{i+1}, x_{i+2}, x_{i+3}\right\}$. Moreover, there is exactly one number divisible by 3 in each subsequent triple of integer numbers, thus after reordering we have $\{i+1, i+2, i+3\}=\{3 s, r, t\}$ for appropriate $r, s$ and $t$, such that $r$ and $t$ are not divisible by 3. Recall that by property (11) at least one of the two monomials $c_{3 s r t} x_{3 s} x_{r} x_{t}$ and $c_{3 s t r} x_{3 s} x_{t} x_{r}$ is different from zero. Hence, the first letters in the leading monomials of the relation $\sum_{\sigma \in S_{3}} c_{\sigma} x_{i+\sigma(1)} x_{i+\sigma(2)} x_{i+\sigma(3)}$ have index divisible by 3 and two remaining letters is not divisible by 3 . Consequently, the leading monomials of generating relations have no compositions and the set of generating relations form a strongly free Gröbner bases following that the algebra $A$ has global dimension 2. (See $[\mathbf{U}] \S 4.3$ for details on strongly free relations.)

1.2. Corollary. The minimal free resolution $\mathbf{A}_{n}$ of the algebra

$$
A_{n}:=\mathbb{k}\left\langle x_{1}, \ldots, x_{n} \mid \begin{array}{l}
{\left[\left[x_{i}, x_{i+2}\right], x_{i+1}\right]} \\
i=1, \ldots, n-2
\end{array}\right\rangle
$$

is generated by $x_{1}, \ldots, x_{n}$ and $u_{1,2,3} \ldots, u_{n-2, n-1, n}$ such that

$$
\begin{array}{cc}
\operatorname{deg}\left(x_{i}\right)=0, & \operatorname{deg}\left(u_{i, i+1, i+2}\right)=-1 ; \\
d\left(x_{i}\right)=0 ; & d\left(u_{i, i+1, i+2}\right)=\left[\left[x_{i}, x_{i+2}\right], x_{i+1}\right] .
\end{array}
$$

Proof. Let us expand the commutators in the relations we are working with:

$$
\left[\left[x_{1}, x_{3}\right], x_{2}\right]=\left(x_{1} x_{3} x_{2}-x_{3} x_{1} x_{2}-x_{2} x_{1} x_{3}+x_{2} x_{3} x_{1}\right)
$$

As we can see they satisfy the condition (11) of Lemma 1.1 and algebra $A_{n}$ has global dimension 2, meaning that the following complex

$$
0 \rightarrow \operatorname{span}\langle\text { relations }\rangle \otimes A_{n} \rightarrow \operatorname{span}\langle\text { generators }\rangle \otimes A_{n} \rightarrow A_{n} \rightarrow \mathbb{k} \rightarrow 0
$$

is acyclic in the leftmost term and, consequently, acyclic everywhere. Therefore, the minimal resolution of $A_{n}$ is generated by generators and generating relations of $A_{n}$. 


\section{REFERENCES}

[B] T. Backman, PhD thesis under preparation.

[CMW] R. Campos, S. Merkulov and T. Willwacher The Frobenius properad is Koszul, arXiv:1402.4048 To appear in Duke Math. J.

[C] M. Chas, Combinatorial Lie bialgebras of curves on surfaces, Topology 43 (2004), no. 3, 543-568.

[CS] M. Chas and D. Sullivan, Closed string operators in topology leading to Lie bialgebras and higher string algebra, in: The legacy of Niels Henrik Abel, pp. 771-784, Springer, Berlin, 2004.

[CFL] K. Cieliebak, K. Fukaya and J. Latschev, Homological algebra related to surfaces with boundary, preprint arXiv:1508.02741 2015.

[D] V. Drinfeld, Hamiltonian structures on Lie groups, Lie bialgebras and the geometric meaning of the classical Yang-Baxter equations, Soviet Math. Dokl. 27 (1983) 68-71.

[ES] P. Etingof and O. Schiffmann, Lectures on Quantum Groups, International Press, 2002.

$[\mathrm{JF}]$ T. Johnson-Freyd, Chains $(\mathbb{R})$ does not admit a geometrically meaningful properadic homotopy Frobenius algebra structure, preprint arXiv:1308.3423 (2013).

[KM] M. Kapranov and Yu.I. Manin, Modules and Morita theorem for operads. Amer. J. Math. 123 (2001), no. 5, $811-838$.

[Ko] M. Kontsevich, a letter to Martin Markl, November 2002.

[MaVo] M. Markl and A.A. Voronov, PROPped up graph cohomology, in: Algebra, arithmetic, and geometry: in honor of Yu.

I. Manin. Vol. II, Progr. Math., 270, Birkhäuser Boston, Inc., Boston, MA (2009) pp. 249-281.

[M1] S.A. Merkulov, Prop profile of Poisson geometry, Commun. Math. Phys. 262 (2006), 117-135.

[M2] S.A. Merkulov, Graph complexes with loops and wheels, in: "Algebra, Arithmetic and Geometry - Manin Festschrift" (eds. Yu. Tschinkel and Yu. Zarhin), Progress in Mathematics, Birkhaüser (2010), pp. 311-354.

[M3] S.A. Merkulov, Lectures on props, Poisson geometry and deformation quantization, in Poisson Geometry in Mathematics and Physics, Contemporary Mathematics, 450 (eds. G. Dito, J.H. Lu, Y. Maeda and A. Weinstein), AMS (2009), 223-257.

[MV] S.A. Merkulov and B. Vallette, Deformation theory of representations of prop(erad)s I 6 II, Journal für die reine und angewandte Mathematik (Crelle) 634, 51-106, \& 636, 123-174 (2009).

[MW1] S.A. Merkulov and T. Willwacher, Props of ribbon graphs, involutive Lie bialgebras and moduli spaces of curves, preprint arXiv:1511.07808 (2015) 51pp.

[MW2] S.A. Merkulov and T. Willwacher, Deformation theory of Lie bialgebra properads, preprint 2015.

[MW3] S.A. Merkulov and T. Willwacher, On explicit formula for quantization of Lie bialgebras, to appear.

[Sc] T. Schedler, A Hopf algebra quantizing a necklace Lie algebra canonically associated to a quiver. Intern. Math. Res. Notices (2005), 725-760.

[Sh] B. Shoikhet, $A L_{\infty}$ algebra structure on polyvector fields, preprint arXiv:0805.3363. (2008).

[U] V. A. Ufnarovsky, Combinatorial and asymptotical methods in algebra, Sovr. probl. mat., Fund. napr., 57 (1990), p. 5-177

[Russian] Engl. transl.: Algebra VI, Encycl. Math. Sci., Springer, Berlin 1995, p. 1-196

[V] B. Vallette, A Koszul duality for props, Trans. AMS, 359 (2007), 4865-4943.

[W1] T. Willwacher, M. Kontsevich's graph complex and the Grothendieck-Teichmüller Lie algebra, Invent. Math. 200 (2015), no. $3,671-760$

[W2] T. Willwacher, The oriented graph complexes, Comm. Math. Phys. 334 (2015), no. 3, 1649-1666.

Anton Khoroshin: National Research University Higher School of Economics, International Laboratory of Representation Theory and Mathematical Physics, 20 Myasnitskay Ulitsa, Moscow 101000, Russia

AND ITEP, Bolshaya Cheremushkinskaya 25, 117259, Moscow, Russia

E-mail address: akhoroshkin@hse.ru

Sergei Merkulov: Mathematics Research Unit, Luxembourg University, Grand Duchy of Luxembourg

E-mail address: sergei.merkulov@uni.lu

Thomas Willwacher: Institute of Mathematics, University of Zurich, Zurich, Switzerland

E-mail address: thomas.willwacher@math.uzh.ch 